\title{
BMJ open Cost-effectiveness of total hip arthroplasty versus resurfacing arthroplasty: economic evaluation alongside a clinical trial
}

\author{
Richard Edlin, ${ }^{1}$ Sandy Tubeuf, ${ }^{2}$ Juul Achten, ${ }^{3}$ Nicholas Parsons, ${ }^{3}$ Matthew Costa ${ }^{4}$
}

To cite: Edlin R, Tubeuf $\mathrm{S}$, Achten J, et al. Costeffectiveness of total hip arthroplasty versus resurfacing arthroplasty: economic evaluation alongside a clinical trial. BMJ Open 2012;2:e001162. doi:10.1136/bmjopen-2012001162

- Prepublication history and additional material for this paper are available online. To view these files please visit the journal online (http://dx.doi.org/10.1136/ bmjopen-2012-001162).

Received 29 March 2012 Accepted 17 August 2012

This final article is available for use under the terms of the Creative Commons Attribution Non-Commercial 2.0 Licence; see http://bmjopen.bmj.com

For numbered affiliations see end of article

\section{Correspondence to} Dr Richard Edlin; r.edlin@auckland.ac.nz

\begin{abstract}
Objective: To report on the relative cost-effectiveness of total hip arthroplasty and resurfacing arthroplasty (replacement of articular surface of femoral head only) in patients with severe arthritis suitable for hip joint resurfacing arthroplasty.
\end{abstract}

Design: Cost-effectiveness analysis on an intention-totreat basis of a single-centre, single-blind randomised controlled trial of 126 adult patients within 12 months of treatment. Missing data were imputed using multiple imputations with differences in baseline quality of life and gender adjusted using regression techniques.

Setting: A large teaching hospital trust in the UK. Participants: A total of 126 adult patients with severe arthritis of the hip joint suitable for a resurfacing arthroplasty of the hip.

Results: Data were received for 126 patients, 4 of whom did not provide any resource use data. For the remainder, data were imputed for costs or quality of life in at least one time point (baseline, 3, 6 months and 1 year) for 18 patients. Patients in the resurfacing arm had higher quality of life at 12 months ( 0.795 vs 0.727 ) and received 0.032 more QALYs within the first 12 months postoperation. At an additional cost of $£ 564$, resurfacing arthroplasty offers benefits at £17 451 per QALY within the first 12 months of treatment. When covariates are considered, the health economic case is stronger in men than in women.

Conclusions: Resurfacing arthroplasty appears to offer very short-term efficiency benefits over total hip arthroplasty within a selected patient group. The shortterm follow-up in this trial should be noted, particularly in light of the concerns raised regarding adverse reactions to metal debris from metal-on-metal bearing surfaces in the longer term. Longer-term follow-up of resurfacing arthroplasty patients and decision analytic modelling is also advised.

Trial registration: Current controlled Trials ISRCTN33354155. UKCRN 4093.

\section{INTRODUCTION}

Hip arthroplasty is acknowledged to be a highly effective and cost-effective procedure for treating patients with severe arthritis of

\section{ARTICLE SUMMARY}

\section{Article focus}

- This article compares the cost-effectiveness of hip resurfacing as against conventional hip arthroplasty surgery.

- The findings are based on data from an RCT conducted within a single, UK centre.

- The analysis considers outcomes within the first year of initial surgery.

Key messages

- Within the first year of treatment, hip resurfacing appears cost-effective.

- The choice of arthroplasty implant appears a key driver of this judgement.

- The evidence for resurfacing appears stronger for male than female patients.

Strengths and limitations of this study

- This article provides the first within-trial data on the cost-effectiveness of resurfacing.

- The period considered here cannot capture important issues (eg, long-term survival of metal-on-metal resurfacing implants versus hip arthroplasty implants).

- The findings merit further analysis and exploration within a decision analytic model.

the hip joint, with $87 \%$ of patients reporting an improvement in their general health following surgery. ${ }^{1}$ The total health gain is expected to be substantial given the effectiveness of treatment; EuroQol (EQ-5D-3L)based quality-of-life improvements following surgery are estimated to be 0.409 , within the 45000 cases measured in the UK Patient Reported Outcomes programme. ${ }^{2}$ A total of $97 \%$ of UK hip replacements are still working (unrevised) at 5 years ${ }^{3}$ and $83 \%$ of all primary hip arthroplasty (all age and all implant types) are unrevised at 17 years postsurgery in Sweden. ${ }^{4}$ If the initial qualityof-life gains are maintained, each unrevised surgery represents over five discounted 
quality-adjusted life-years (QALYs) gained and a benefit of over 100000 pounds at the $£ 20000$ per QALY threshold used by the National Institute of Health and Clinical Excellence (NICE). Compared to these gains, the costs of hip arthroplasty surgery appear modest. As a result, most analyses considering health economics have concentrated on questions of which type of prosthesis to use, and many cost-effectiveness analyses have involved analysis of newer, more expensive operations against older, established comparators. ${ }^{5-7}$ Resurfacing arthroplasty (RSA) of the hip is a newer alternative form of arthroplasty designed for younger, active patients with severe arthritis of the hip.

Hip RSA involves the insertion of an acetabular component and the 'capping' of the femoral neck, rather than its removal and replacement with a femoral component in a standard total hip arthroplasty (THA). Of the 70000 hip arthroplasty operations conducted in England and Wales every year, ${ }^{3}$ approximately $6 \%$ are hip resurfacings. The equivalent figure among men aged under 55 is $33 \%$. As resurfacing preserves the bone of the proximal femur, it may be expected to provide better clinical outcomes on revision of this component than available with a standard hip arthroplasty. Despite advances in their construction, there are still questions about the durability of modern resurfacing implants and there have been few explicit economic evaluations comparing resurfacing arthroplasties against total hip arthroplasties. ${ }^{8} 9$ Few randomised controlled trials have been conducted to assess the outcomes of hip resurfacing, and those that exist provide little detail about the economic costs and benefits within the initial year following surgery. This paper reports the first within-trial economic evaluation of RSA versus THA.

\section{METHODS}

\section{Interventions and sample}

This evaluation reports on the efficiency of RSA versus THA. Patients were deemed eligible for the trial if they were aged over 18 years of age, were medically fit for an operation and were deemed suitable to receive an RSA. Patients were only excluded from the study if there was evidence that the patient would be unable to adhere to trial procedures or complete questionnaires. Patients were randomised on a 1:1 basis between THA and RSA, with each patient operated on according to the preferred technique of the operating surgeon. Other perioperative interventions, such as prophylactic antibiotics and thrombo-prophylaxis were the same for all patients and the same standardised rehabilitation plan was employed for both trial arms. Further details on recruitment, ethics and randomisation procedures are reported in both the randomised controlled trial's (RCT) protocol and reporting papers. ${ }^{10} 11$ The main outcome measure of the trial was hip function (Oxford Hip Score; Harris Hip Score) at 12 months, and the trial found no evidence of a difference between RSA and THA.

\section{Perspective}

The aim of the economic study is to determine the intervention that would maximise health outcomes within the limited National Health Service (NHS) budget in this period, and so a cost-effectiveness (cost-utility) analysis with an NHS and Personal Social Services (PSS) perspective is adopted in the base case. This paper considers the within-trial period (as intention to treat) of the first 12 months of follow-up. It considers only resources used within the NHS setting including any aids and adaptations required. The base year for all costs figures was 2009/2010, with figures from other years converted using the hospital and community health services Pay and Prices Index (for adults, excluding capital). ${ }^{12}$ For current costs, figures are deflated assuming an estimated inflation rate of $1.9 \%$ to 2010 from this index for both 2009/2010 and 2010/2011. As the analysis uses a 1-year time horizon, discounting for the future cost and health outcome is not necessary in this analysis. The currency used was the pound sterling (£).

\section{Quality of life}

Responses from the EQ-5D-3L were obtained from patients at baseline, 3, 6 and 12 months, respectively, as secondary outcomes of the trial ${ }^{10}$; results from other outcomes are reported in greater depth elsewhere. ${ }^{11}$ The standard tariff values ${ }^{13}$ were applied to these responses at each time point to provide EQ-5D-3L quality-of-life values. QALYs were calculated as an 'area under the curve' and form the main outcome measure of the study. Where comparisons between the RSA and THA arms are based on non-imputed data, a two-sample $\mathrm{t}$ test assuming equal variances is used.

\section{Resource use and valuation}

The costs of THA and RSA treatments were considered across six broad categories - the costs of the initial operation, of inpatient care postdischarge, of outpatient care, of primary/community care, and of medications, and aids/adaptations required while in the community. The analysis considered inpatient and outpatient attendances for all reasons, and requested details of other resource usage only where it related to pain or hip surgery.

All RSA patients received a Cormet metal-on-metal (MOM) resurfacing (Corin Group, Cirencester, UK), while THA patients received their surgeon's preference of prosthesis. For the patients having THA the prosthesis type was identified from patient records, with three types of bearing surface (ceramic femoral head on ceramic socket, MOM and metal-on-polyethylene) accounting for 95\% of cases. The University Hospitals Coventry and Warwickshire NHS Trust Finance Department provided implant list prices for both the resurfacing implant and representative cost figures for these three types of prosthesis. In the remaining $5 \%$ of cases, implant type was treated as missing and were imputed to fall in one of these groups. 
The current Healthcare Resource Group v.4 (HRG4) reference costs include the cost of prosthesis across all ages, and in most cases this will be a THR as HRG4 does not include a single category for primary replacements (as appeared in previous versions). Identified nationallevel HRG4 frequencies for primary hip replacements are available $^{14}$ and these are used to calculate an average cost, average length of stay and average cost per excess bed day. By deducting the expected THA cost from the average cost, we obtain a non-prosthesis average cost, to which it is possible to add the appropriate prosthesis cost relevant to each individual. From here, an average cost of the initial hospitalisation is calculated for each patient by adjusting for each patient's length of stay (as a number of bed days from the mean). In this way, a person admitted for the average length of stay would be assigned the average cost of treatment, with those staying shorter and longer periods assigned lower and higher costs, respectively.

Data regarding length of stay and implant received were obtained from hospital records, with the remainder of the costing information obtained from patient-reported data. Resource usage was assessed alongside other outcomes at 3, 6 and 12 months, respectively. For the 3-month data, the recall period was since discharge from hospital. For the other cases, it was since the last questionnaire was due to be completed. The questionnaires included sections on further inpatient care following the initial operation (specialty and length of stay/day case), outpatient care, primary and community care, aids and adaptations provided by the NHS/social services and medication (pain relief and other NHS medication). Medicines usage was estimated based on mean dosage when used and average usage within the three budgetary periods (discharge to 3 , 3-6 and 6-12 months, respectively). In order to convert resource usage figures into costs, unit cost figures were assigned from NHS reference costs, ${ }^{15}$ Personal Social Services Research Unit (PSSRU) unit costs, ${ }^{12}$ NHS electronic drug tariff ${ }^{16}$ and reported unit costs of acupuncture and chiropractic from previous studies. Individual resource items and unit prices, including for aids and adaptations, are available in Tables provided as a Web Extra. Where statistical tests analyse resource usage data, $\mathrm{t}$ tests are used to test for differences in expected usage (assuming equal variance and non-imputed data).

Data on personal costs (out of pocket medicine usage and time off work for either the patient or a carer) were also collected. NHS unit costs were used to provide an indicative figure for private medicines costs, while 2009 median gross weekly earnings from full time jobs (£488.70) was used to identify a daily productivity cost of $£ 97.74$. These are used in the sensitivity analysis considering societal costs.

\section{Missing data}

Where data were incomplete we used multiple imputation via chained equations (ice) ${ }^{17}$ to complete missing data using STATA V.11 (StataCorp 2009, Texas,
USA). ${ }^{18}{ }^{19}$ Missing cost data were predicted in terms of QALYs, treatment received, length of stay, age, gender, height, weight and baseline clinical scores (Oxford Hip Score, Harris Hip Score); missing QALY data were predicted in terms of this same list (excluding QALYs), plus each of the cost items; missing length of stay was predicted using the same list as for QALYs, with QALYs included. In order to remove implausible data, missing cost data were constrained to be positive and length of stay was constrained to be at least three days postimputation. A total of 50 imputations were used to inform each item of missing data. Where tests are conducted to detect significant differences in mean values between the RSA and THA groups based on imputed data (ie, incremental costs and QALYs), the analysis uses an OLS regression within the STATA's mim command.

\section{Cost-effectiveness}

Using the methods identified above, total costs and QALY figures were calculated for all patients including imputed data. For the cost-effectiveness analysis, we identified the differences between costs and QALYs between the two arms, dividing the former by the latter to compute an incremental cost-effectiveness ratio (ICER). When compared against the marginal trade-off for the NHS as a whole-the cost-effectiveness threshold-the ICER gives a broad indication of whether spending additional money on hip arthroplasty appears efficient. The ICER figure is not presented with a CI due to difficulties in interpreting a ratio of two random variables. Instead, we assume that each QALY is valued at $£ 20000$ and subtract costs from this 'monetised' QALY in order to obtain a net monetary benefit (NMB). Any treatment with an ICER below $£ 20000$ will have a positive NMB, with higher NMB figures unambiguously better and lower NMB figures unambiguously worse. As before, a 95\% CI is formed for NMB using linear regression using STATA's mim command.

\section{Scenarios/univariate sensitivity analyses}

Key uncertainties in the scenarios considered were explored using univariate sensitivity analyses. The results for complete cost and quality-of-life data (ie, those with no missing data) were provided to identify the impact of missing data on the analysis. A strict per-protocol analysis of the data is also used to reflect any sensitivity to protocol violations. A societal perspective was also explored by adding the patient medicines and productivity costs outlined above to the NHS+PSS costs. As patients might also recover function within the first 3 months (rather than continuously to 3 months), a quicker initial recovery was explored in QALY calculations, where each patient's quality of life was assumed to reach its observed 3-month level at 6 weeks postoperatively. The cost assumptions in the analysis were modified by assessing the impact of assuming the least expensive (metal on polyethylene) THA implant was used throughout with no effect on observed outcomes, to reflect the potential 
concern that the THA arm might not reflect costeffective practice. The recent (after the trial) current recommendations against the use of metal-on-metal THA prostheses are briefly considered by setting all 'metal-on metal' implants to missing, estimating which THA prosthesis (ie, metal on polyethylene or ceramic on ceramic) each patient will receive using multiple imputation, and considering the cost implications within these alternative estimates.

\section{Adjustment for potential baseline differences}

The base-case analysis was conducted to allow for comparability between this within-trial analysis and the reporting of the main RCT. ${ }^{11}$ These quality-of-life and gender-based analyses are conducted as sensitivity analyses to allow comparability with the main RCT, which did not find a significant difference in baseline quality of life and did not test for an interaction between efficacy and gender. Given that these issues may be important within the economic evaluation, they are considered as sensitivity analyses.

The impact of potential baseline differences in quality of life is corrected for using regression analysis within a sensitivity analysis. The number of QALYs received (average quality of life over 12 months) is assumed to be a normal distribution, conditional on trial arm (RSA or THA) and baseline EQ-5D-3L value. Total cost over 12 months is assumed to be lognormal, so that the natural logarithm of costs is a normal distribution, conditional on trial arm, baseline EQ-5D-3L.

QALYs and (log-) costs for each person are estimated using ordinary least-squares regression (using STATA's mim command to handle imputed data). As any relationship between uncertainty in the extra costs and benefits associated with RSA is important when assessing the likelihood of cost-effectiveness, we use a seemingly unrelated regression to do this. By using a Cholesky decomposition of the variance-covariance matrix, (log-) costs and QALYs are modelled as if they come from a multivariate normal distribution. Uncertainty in the value of other items in the regression is ignored. From here, costs are estimated as if all patients receive THA, and incremental costs are calculated as a proportion of the average THA cost. In this way, a distribution is built up for incremental costs and incremental QALYs that can be analysed using cost-effectiveness acceptability curve (CEAC) can be formed for this analysis. ${ }^{20}$ This CEAC indicates the likelihood that RSA will be cost-effective at different 'values' for a QALY.

As gender so heavily affects the clinical use of RSA, this analysis was rerun for both male patients only and female patients only. This allows the effects of RSA to be assessed separately for men and women, with this figure presented as the likelihood of that RSA would be costeffective at a threshold value of $£ 20000$ per QALY.

\section{RESULTS}

\section{Trial recruitment}

The trial $^{11}$ recruited a total of 126 patients $(\mathrm{RSA}=60$; THA=66) between May 2007 to February 2010. Two patients from each arm of the study did not have surgery and provided only baseline quality-of-life/demographic data, leaving a total of 58 and 64 patients in each arm. The sample was representative of the broader population undergoing resurfacing in the UK during the period of recruitment; no significant differences were identified between those who took part and those who were eligible but chose not to take part. Further details on both the ethical approval for the study and the demographics of the patients are provided in the clinical paper. ${ }^{11}$ As the analysis estimates data on costs and outcomes conditional on baseline quality of life, these patients cannot contribute any data to our analysis and are excluded from the analyses here.

\section{Quality of life}

Table 1 summarises quality-of-life estimates at the four time points and calculates QALY estimates both with and without data imputation in the two arms. Overall, those in the RSA group started in worse health (as measured by the EQ-5D-3L) and received 0.033 more QALYs within the 12 months of the trial ( $n=118$ observations). When the small amount of missing data is imputed, the estimated benefit remains very similar at 0.032 (95\% CI -0.054 to 0.119$)$. Within the trial, the difference in quality of life between the RSA and THA arms of the trial appears to increase at each postoperative time point.

Table 1 EQ-5D-3L quality of life at each measurement and converted into quality-adjusted life-years (QALYs) (missing data imputed)

\begin{tabular}{lllr}
\hline Quality of life & RSA (SD) $\mathbf{n = 5 8}$ & THA (SD) $\mathbf{n = 6 4}$ & Difference (95\% CI) \\
\hline Baseline & $0.308(0.338)$ & $0.356(0.335)$ & $-0.048(-0.168$ to 0.073$)$ \\
3 months & $0.722(0.229)$ & $0.698(0.284)$ & $0.023(-0.711$ to 0.118$)$ \\
6 months & $0.796(0.244)$ & $0.747(0.287)$ & $0.050(-0.046$ to 0.146$)$ \\
12 months & $0.795(0.282)$ & $0.727(0.319)$ & $0.067(-0.042$ to 0.177$)$ \\
QALYs $(n=118)$ & $0.716(0.216)$ & $0.683(0.252)$ & $0.033(-0.053$ to 0.120$)$ \\
QALYs* $(n=122)$ & $0.713(0.216)$ & $0.681(0.251)$ & $0.032(-0.054$ to 0.119$)$ \\
\hline${ }^{*}$ With imputed data. & &
\end{tabular}




\section{Costs and resource usage}

Overall, NHS and social care costs were significantly higher among the RSA group with an average of $£ 564$ more spent within the first 12 months from the operation (table 2), of which the majority is due to the higher cost of implants and length of stay following the initial operation (£184), subsequent inpatient care (£279) and outpatient care (£84). The deflated cost of the RSA implants including operative consumables used in this study was $£ 1826$ versus an average of $£ 1700$ for THA operations, based on imputed data. THA implants differed in costs, with the most expensive being ceramic-on-ceramic implants (£2042) and those using metal-on-metal implants costing slightly less than RSA implants (£1625). Implants and consumables in metal-on-polyethylene operations (£843) were associated with only $40 \%$ of the cost of ceramic-on-ceramic implant. While the resurfacing implants were more expensive, they were also associated with a slightly longer length of stay (5.7 versus 5.5 days), although this difference was not statistically significant $(\mathrm{p}=0.536$; imputed data).

Those in the RSA arm had significantly more outpatient visits than those in the THA arm (5.155 versus 3.063, $\mathrm{p}=0.0054$; non-imputed data). Here, both the number of physiotherapy sessions and the use of deep vein thrombosis assessments were significantly higher among this group $(\mathrm{p}=0.002, \mathrm{p}=0.011$; non-imputed data). For inpatient care, only subsequent inpatient attendances $(0.155$ versus $0.047, \mathrm{p}=0.066$; non-imputed data) approached significance, with the only significant difference $(p=0.009)$ in aids and adaptations favouring RSA. For full details on individual resource use items and their unit costs, please see the tables available as a Web Extra.

The private costs to patients following arthroplasty surgery are considerable, although relatively little of this is due to the purchase of medication. There are no significant differences in medication usage between the RSA and THA arms, and the total costs of this treatment is similar ( $£ 12$ RSA versus £9 THA, $\mathrm{p}=0.667$ ). RSA patients report an average of 73 days off work, as against 57 days for THA patients $(\mathrm{p}=0.333)$. While surgery results in a large number of days off work for the patient, carers tend to take very few days off work (2.1 days RSA versus 1.6 days THA; $\mathrm{p}=0.595$ ). Overall, RSA patients report costs valued at £5917, as against $£ 5853$ in the THA arm (imputed data). This difference is small but highly uncertain, such that there is no significant difference in costs from a societal perspective ( 629 higher costs in RSA, 95\% CI -£2456 to £3713).

\section{Cost-effectiveness and sensitivity analyses}

While RSA is expected to cost more over the first 12 months following an operation, it appears to provide a difference in quality of life. Here, the incremental ICER for RSA is $£ 17451$ per QALY ( $£ 564 / 0.032$ QALY). Within most of the sensitivity tests explored here, the figure appears to remain within or below the $£ 20 \mathrm{k}-£ 30 \mathrm{k}$ per QALY range used by the National Institute for Health and Clinical Excellence as its estimate of the cost-effectiveness threshold, except where cheaper THA implants are used in place of surgeon's preference, which was mostly MOM THA within the trial (table 3 ). If the cheaper (metal-on-polyethylene) implants are used, the increased cost of RSA versus THA implants is enough to raise the average cost difference above $£ 1000$ which, given the small quality-of-life difference observed here, is enough to prevent RSA being cost-effective. However, if we consider both types of non-MOM implants (ceramic-on-ceramic and metal-on-polythene), this difference disappears entirely as the non-MOM implants were slightly more expensive on average than the MOM ones. The CI for net benefit in every analysis spans zero (table 4) so that the findings do not reach statistical significance. As clinical trials are very rarely designed with the power of cost-effectiveness conclusions in mind, very little can be inferred from this lack of significance.

\section{Adjustment for baseline differences}

Once baseline differences in EQ-5D-3L are considered, the QALY estimates for the first 12 months appear to change. QALYs are higher generally among those who are healthier at baseline (EQ-5D-3L; $\mathrm{p}=0.000$ ), with those treated in the RSA arm receiving 0.053 more QALYs than those treated with THA $(\mathrm{p}=0.119)$. Likewise, $(\log )$-costs

Table 2 Costs by type, summed across trial period (missing data imputed)

\begin{tabular}{lccrr}
\hline Costs & \% Imputed & RSA (SD) $\mathbf{n = 5 8}$ & THA (SD) $\mathbf{n = 6 4}$ & Difference (95\% CI) \\
\hline Initial operation/care & 7 & $£ 6275(557)$ & $£ 6091(532)$ & $£ 184(-18$ to 386) \\
Subsequent inpatient & 11 & $£ 470(956)$ & $£ 191(558)$ & $£ 279(-11$ to 569) \\
Outpatient & 11 & $£ 360(294)$ & $£ 276(210)$ & $£ 84(-13$ to 181) \\
Primary/community & 11 & $£ 63(98)$ & $£ 49(67)$ & $£ 14(-17$ to 45) \\
Aids and adaptations & 11 & $£ 21(33)$ & $£ 21(40)$ & $£ 0(-14$ to 14) \\
Medication & 11 & $£ 27(43)$ & $£ 24(41)$ & $£ 3(-13$ to 19) \\
NHS+PSS costs & - & $£ 7217(1320)$ & $£ 5653(917)$ & $£ 564(144$ to 985) \\
Private costs & 61 & $£ 5917(5145)$ & $£ 553(5520)$ & $£ 64(-3017$ to 3146) \\
Societal cost & - & $£ 13134(5146)$ & $£ 12506(5568)$ & $£ 629(-2456$ to 3713) \\
\hline NHS, National Health Service; PSS, Personal Social Services; RSA, resurfacing arthroplasty; THA, total hip arthroplasty.
\end{tabular}


Table 3 Incremental cost effectiveness

\begin{tabular}{lclr}
\hline Scenario & Incremental costs (95\% CI) & Incremental QALYs (95\% CI) & ICER (per QALY) \\
\hline Base case & $£ 564(144$ to 985$)$ & $0.032(-0.054$ to 0.119$)$ & $£ 17451$ \\
Per protocol & $£ 528(85$ to 970$)$ & $0.024(-0.066$ to 0.113$)$ & $£ 22227$ \\
Complete case data (N=98) & $£ 721(286$ to 1157$)$ & $0.053(-0.042$ to 0.149$)$ & $£ 13443$ \\
Societal costs & $£ 629(-2456$ to 3713$)$ & $0.032(-0.054$ to 0.119$)$ & $£ 19435$ \\
Metal/polyethylene THA implants & $£ 1271(859$ to 1684$)$ & $0.032(-0.054$ to 0.119$)$ & $£ 39318$ \\
No metal-on-metal THA implants & $£ 522(76$ to 968$)$ & $0.032(-0.054$ to 0.119$)$ & $£ 16137$ \\
Quicker initial recovery & $£ 564(144$ to 985$)$ & $0.039(-0.048$ to 0.127$)$ & $£ 14310$ \\
QoL adjustments & $£ 473(113$ to 853$)$ & $0.053(-0.014$ to 0.120$)$ & $£ 8905$ \\
QoL adjustments, males only & $£ 402(-82$ to 916$)$ & $0.073(-0.012$ to 0.158$)$ & $£ 5519$ \\
QoL adjustments, females only & $£ 598(64$ to 1172$)$ & $0.037(-0.070$ to 0.144$)$ & $£ 16272$ \\
\hline ICER, incremental cost-effectiveness ratio; QoL, quality-of-life; THA, total hip arthroplasty. &
\end{tabular}

appear to be affected by baseline health $(\mathrm{p}=0.034)$, with costs $7.1 \%$ higher (95\% CI $1.7 \%$ to $12.9 \%$ ) for those who received RSA after bootstrapping.

While correcting for baseline differences leaves the incremental costs largely unchanged (£473; 95\% CI: 107 to 840$)$, the estimated QALY benefit almost doubles (0.053, 95\% CI: -0.014 to 0.120$)$. Consequently, the ICER is around half as large ( $£ 8905$ per QALY) as the non-adjusted case. In $79 \%$ of cases investigated, RSA is recommended when valuing health at $£ 20000$ per QALY - suggesting quite high confidence that RSA is the more cost-effective option within the first 12 months of treatment across the $£ 20 \mathrm{k}-£ 30 \mathrm{k}$ range used by NICE (figure 1). Where this analysis is rerun for male patients only $(n=71)$, neither incremental costs nor incremental QALYs reach statistical significance and the ICER falls to $£ 5519$ per QALY. For female patients $(n=51)$, the ICER is about three times as large as for males (£16 272 per QALY) due to higher costs and lower benefits, with the latter exacerbated by a much lower baseline quality of life (female 0.257, male 0.389; $\mathrm{p}=0.032$ ). Within the scenarios used here, RSA is only $54 \%$ likely to be cost-

Table 4 Net monetary benefit

\begin{tabular}{lr}
\hline Scenario & NMB (95\% Cl)* \\
\hline Base case & $£ 82.46(-1795$ to 1960) \\
Per protocol & $-£ 53(-2011$ to 1905) \\
$\begin{array}{l}\text { Complete case data (N=98) } \\
\text { Societal costs }\end{array}$ & $£ 353(-1719$ to 2426) \\
Metal/polyethylene THA & $-£ 625(-2515$ to 1265) \\
implants & \\
No metal-on-metal THA implants & $£ 125(-1750$ to 1999) \\
$\begin{array}{l}\text { Quicker initial recovery } \\
\text { Adjustments for quality of life }\end{array}$ & $£ 224(-1658$ to 2107) \\
$\begin{array}{l}\text { Adjustments for quality of life, } \\
\text { males }\end{array}$ & $£ 1055(-843$ to 2014) \\
$\begin{array}{l}\text { Adjustments for quality of life, } \\
\text { females }\end{array}$ & $£ 137(-1988$ to 2262) \\
\hline $\begin{array}{l}\text { QALYs valued at £20k each. } \\
\text { NMB, net monetary benefit, THA, total hip arthroplasty. }\end{array}$
\end{tabular}

effective for female patients at $£ 20000$ per QALY, compared to an $86 \%$ likelihood for male patients.

\section{DISCUSSION}

In comparison with standard THA, hip RSA appears to provide a modest QALY gain for a modest sum within the first 12 months from surgery; while the additional costs of RSA are statistically significant, the additional benefits are not. The higher costs of RSA treatments are largely due to slightly higher costs for the initial operative and recovery periods, and higher usage of outpatient services. While the RSA group achieves slightly better health outcomes and requires more services, this may be due to heterogeneity in outcomes; if resurfacing works well for most but poor for some, then this could produce this type of phenomenon. If so, this emphasises the need to follow patients up in the longer term.

The analysis presented here analyses the data by considering potential confounding due to both gender and baseline quality of life, and this nearly doubles the estimate of RSA effect size. While the main analysis of the trial data ${ }^{11}$ found no statistically significant difference in hip function between the RSA and THA groups at 12 months, it seems likely that some short-term difference in quality of life exists favouring RSA and that-again within 12 months-there is enough evidence to suggest that it may be cost effective.

Within the first 12 months of treatment, the main caveat to our results deals with the comparator THA arm. The pragmatic nature of the trial data used here ${ }^{11}$ is one of its key strengths, since it reflects current practice. Any changes to this practice may affect cost-effectiveness though, so that RSA may become more/less cost-effective as less/more cost-effective THA implants are used. A recent (US) analysis of registry data suggests that more expensive implants do not provide a substantive age-adjusted advantage over less expensive prostheses. ${ }^{21}$ Where the sensitivity analysis assumed the use of the cheapest metal-on-polyethylene implants (without incorporating a possible impact on quality of life), RSA was no longer cost-effective within-trial. However, these implants were used relatively rarely in practice, and the 


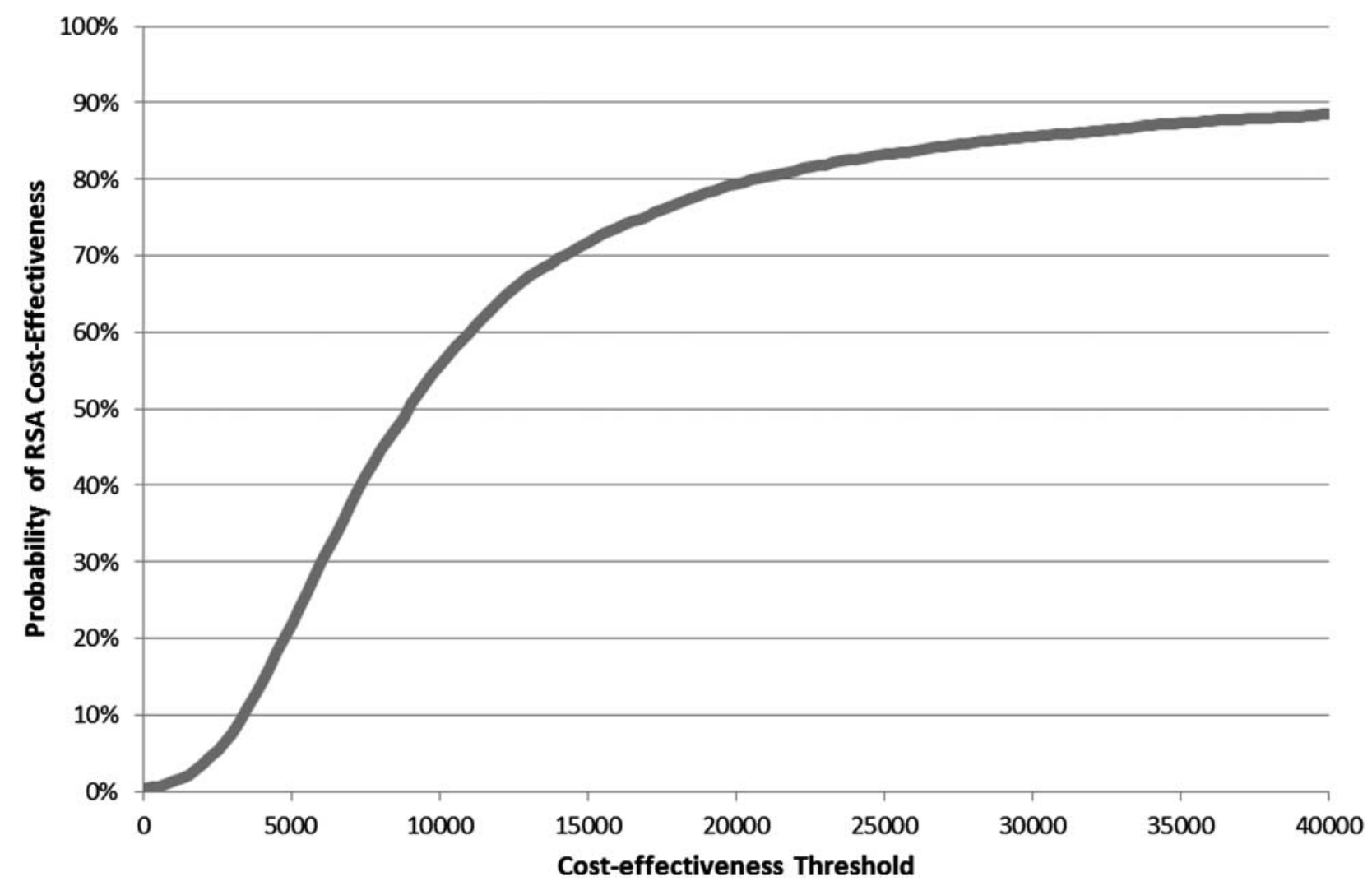

Figure 1 Cost-effectiveness acceptability curve for resurfacing arthroplasty

main alternative to metal-on-metal THA implants was the more expensive ceramic on ceramic type. Restrictions in the use of MOM THA implants within the UK are likely to lead to more costly THA implants being used, and so a net increase in the cost-effectiveness of resurfacing by comparison.

Beyond the issues surrounding the choice of THA, the trial is inevitably unable to consider all possible cost items. The trial did not explicitly consider any differences in operative time between the RSA and THA arms; no difference was expected and an informal analysis of the data suggests very similar operative times between the arms. This evaluation was also unable to consider the impact of variation in cost within each type of prostheses (ie, within the three types of THA, or beyond the single RSA used in the trial) as this information is not generally available. The clinical trial upon which this analysis is based used a single type of Cormet prosthesis that has been used in the UK for around 15 years. While the list price of the Cormet prosthesis is similar to other prostheses available locally, prices are hospital specific and so some caution is warranted when seeking to generalise findings to other locations. We note also that our findings are not necessarily generalisable to other types of resurfacing, including emerging technologies such as ceramic-on-ceramic resurfacings. While the cost-effectiveness of these newer treatments may differ from standard resurfacings, we cannot identify the most cost-effective type of resurfacing as this was beyond the scope of the trial and relatively little data exists on which to base even a preliminary estimate. To the degree that this may prove possible, it is an issue for subsequent decision analytic modelling.

Clearly, the cost-effectiveness of resurfacing is likely to require assessment over a longer period of time-as is typically the case for any health economic analysis of trial data. ${ }^{22}$ Importantly, the higher revision rates reported for RSA suggest that the additional costs of RSA may be higher if a longer period is considered. On the benefit side of the equation, the impact of extending the time period is unclear as RSA may improve quality of life in the short term but lead to a quicker deterioration once revisions are necessary, or require additional monitoring or revisions by virtue of its 'MOM' nature. One method to explore these questions may be decision analytic modelling. ${ }^{22}$ The trial provides an estimate of short-term clinical benefits from hip function and quality of life (conditional on EQ-5D-3L), with longer follow-up series (from trials or registry data) needed to model implant survival for both RSA and THA.

As THA revision surgery may be surgically more complex, financially more costly and less effective than a primary THA, a key question when interpreting this study is the prognosis for patients after their RSA is revised. An Australian registry analysis suggests poor implant survival among patients receiving a revision of only the acetabular RSA component, and some evidence of higher revision risks among other types of RSA revisions such as where both components are revised. ${ }^{23}$ It is 
unclear, however, as to whether a revised RSA is more similar, in terms of quality of life, to a primary THA or a revision THA. Further research is necessary to assess the likely impact of this and other questions to guide future research, and the findings of this paper are by no means a complete answer to the decision problem.

Registry data reveal that women represent $61 \%$ of primary THA patients in the UK but make up only $25 \%$ of RSA patients. ${ }^{3}$ These figures reflect relevant gender differences from both a clinical and a health economic perspective as women appear to obtain higher quality-of-life gains from THA, and face an increased revision rate from RSA. ${ }^{42}$ This trial may also suggest a lower benefit from RSA relative to THA among women, although the finding was not statistically significant (or powered to be so). Despite the conclusions of the within-trial analysis, it seems clear that until such work is done and further data are available, the cost-effectiveness of RSA in the UK context remains potentially promising but as yet unproven.

\section{Author affiliations}

${ }^{1}$ Health Systems, School of Population Health, University of Auckland, Auckland, New Zealand

${ }^{2}$ Academic Unit of Health Economics, University of Leeds, Leeds, UK ${ }^{3}$ Division of Health Sciences, University of Warwick, Coventry, UK ${ }^{4}$ Warwick Clinical Trials Unit, University of Warwick, Coventry, UK

Contributors RE designed the health economic analysis, with input from MC. ST managed data entry, with both RE and ST conducting elements of the health economic analysis. All authors were responsible for writing the manuscript. All authors read and approved the final manuscript.

Funding The work described in this manuscript has been funded through the Research for Patient Benefit scheme of the NIHR, grant number PB-PG-0706-10080. This manuscript presents independent research commissioned by the National Institute of Health Research. The views expressed are those of the authors and not necessarily those of the NHS, the NIHR or the Department of Health.

Competing interests All authors declare grant funding via the NIHR. In addition, MC and NP declare that manufacturers of resurfacing and total hip replacements have paid research grants to their host institutions, but not in relation to this work.

Data sharing statement No additional data are available.

\section{REFERENCES}

1. Health Episodes Statistics Online. Finalised PROMs data 2009-10, 2011.

2. Health Episodes Statistics Online. PROMs Score Comparisons April 2009 to February 2011: The NHS Information Centre for Health and Social Care, 2011.
3. Ellams D, Forsyth O, Mistry A, et al. 7th Annual Report. National Joint Registry for England and Wales; 2010.

4. Garellick G, Kärrholm J, Rogmark C, et al. Swedish Hip Arthroplasty Register: Annual Report 2008. Shortened Version. Department of Orthopaedics, Sahlgrenska University Hospital; 2009.

5. Bozic KJ, Morshed S, Silverstein MD, et al. Use of cost-effectiveness analysis to evaluate new technologies in orthopaedics: the case of alternative bearing surfaces in total hip arthroplasty. J Bone Joint Surg 2006;88:706-14.

6. Briggs A, Sculpher M, Dawson J, et al. Modelling the cost-effectiveness of primary hip replacement: how cost-effective is the Spectron compared to the Charnley prosthesis? CHE Technical Paper Series 28. York: University of York, 2003:51.

7. Fitzpatrick R, Shortall E, Sculpher $M$, et al. Modelling of cost-effectiveness of THR: methods and results and discussion in primary total hip replacement surgery: a systematic review of outcomes and modelling of cost-effectiveness associated with different prostheses. Health Technol Assess 1998;2:17-32.

8. Vale L, Wyness L, McCormack K, et al. A systematic review of the effectiveness and cost-effectiveness of metal-on-metal hip resurfacing arthroplasty for treatment of hip disease. Health Technol Assess 2002;6(15)

9. Bozic KJ, Pui CM, Ludeman MJ, et al. Do the potential benefits of metal-on-metal resurfacing justify the increased cost and risk of complications? Clin Orthop Relat Res 2010;468:2301-12.

10. Achten JA, Parsons NR, Edlin RE, et al. A randomised controlled trial of total hip arthroplasty versus resurfacing arthroplasty in the treatment of young patients with arthritis of the hip joint. $B M C$ Musculoskelet Disord 2010;11(8).

11. Costa ML, Achten J, Parsons NR, et al. Total hip arthroplasty versus resurfacing arthroplasty in the treatment of young patients with arthritis of the hip joint: a single centre, parallel group, assessor blind, randomised control trial. BMJ 2012;344:e2147.

12. Curtis L. Unit Costs of Health \& Social Care 2010. Personal and Social Services Research Unit, 2010:1-253.

13. Dolan P. Modeling valuations for EuroQol health. Med Care 1997;35:1095-108.

14. HRG version 3.5 \& HRG4 Comparative Chapter Analysis: The Health \& Social Care Information Centre, 2008.

15. Department of Health. National Schedule of Reference Costs 2009-10. Appendix NSRC04: NHS Trusts and PCTs combined reference cost schedules. London: Crown Copyright, 2011.

16. NHS. Electronic Drug Tariff: May 2011. National Health Service England and Wales, 2011.

17. White IR, Royston P, Wood AM. Multiple imputation using chained equations: issues and guidance for practice. Stat Med 2011;30:377-99.

18. Royston P. Multiple imputation of missing values: further update of ice, with an emphasis on interval censoring. Stata $J$ 2007;7:445-64.

19. Royston P, Carlin JB, White IR. Multiple imputation of missing values: new features for mim. Stata J 2009;9:252-64.

20. Fenwick E, Byford S. A guide to cost-effectiveness acceptability curves. Br J Psychiatry 2005;187:106-8.

21. Gioe TJ, Sharma A, Tatman $P$, et al. Do 'premium' joint implants add value? Clin Orthop Relat Res 2011;469:48-54.

22. Petrou S, Gray A. Economic evaluation using decision analytical modelling: decision, conduct, analysis, and reporting. BMJ 2011;342. doi: 10.1136/bmj.d766

23. de Steiger RN, Miller LN, Prosser GH, et al. Poor outcome of revised resurfacing hip arthroplasty. 397 cases from the Australian Joint Replacement Registry. Acta Orthop 2010;81:72-6.

24. Kärrholm J, Garellick G, Rogmark C, et al. Swedish Hip Arthroplasty Register: Annual Report 2007. Department of Orthopaedics, Sahlgrenska University Hospital, 2008. 